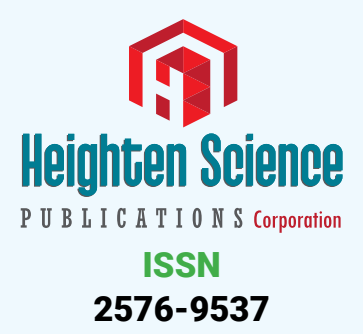

*Address for Correspondence: Luisetto $M$, European Specialist in Laboratory Medicine, Hospital Pharmacist's Manager, Independent researcher, Italy, Email: maurolu65@gmail.com

Submitted: 01 August 2017

Approved: 17 August 2017

Published: 18 August 2017

Copyright: 이 2017 Luisetto $\mathrm{M}$, et al. This is an open access article distributed under the Creative Commons Attribution License, which permits unrestricted use, distribution, and reproduction in any medium, provided the original work is properly cited

Keywords: Surgery; Atherosclerotic disease; Pharmacology; Drug delivery systems; Cardiology; Cardiac surgery; CHOF
Review Article

\section{Surgery and new Pharmacological strategy in some atherosclerotic chronic and acute conditions}

\author{
Luisetto $\mathrm{M}^{1 *}$, Nili-Ahmadabadi $\mathrm{B}^{2}$ and Ghulam Rasool Mashori ${ }^{3}$ \\ 'European Specialist in Laboratory Medicine, Hospital Pharmacist's Manager, Independent researcher, Italy \\ ${ }^{2}$ Nano Drug Delivery, Chapel Hill, NC, USA \\ ${ }^{3}$ Professor \& Director, Peoples University of Medcial \& Health Sciences for Woman, Nawabshah, Pakistan
}

\section{Introduction}

In actual pharmacological therapy we can see that some drugs can be added to other medical instruments to improve their activity: in example we can see medicated stent for some coronary disease, or hormonal medical devices used in pregnancy prevention, but other example are known today. In example Carmustine wafer is delivered by delivery systems in some brain cancer and radioactive seed implants in prostatic cancer. Ocular intra vitreal implants for some macular degenerations (MABS or cortisones) other implants delivery systems drugs, naltrexone implant for opiate dependence. Other strategies imply carrier use to deliver the drugs in the site of action: In example MABS linked to radioactive isotopes in some relapse of severe Hodgkin disease but many other example we can see in therapy used today. So we can think that other chronic conditions can be treated using a combination of drugs with other instrument to improve the clinical outcomes. This to make possible that the ERLICH MUGIC BULLETS can act in the right site reducing the side effect. In example today we can see various medical interventional radiological strategy to treat in coronary and hearth disease with medicate stents positioning or to local use of contrast agents or other valvle surgery procedures with global good clinical results.

\section{Material and Methods}

In this observational work we analyze some relevant (in our opinion) publications on PubMed in order to evaluate actual option available today in some heart disease and we try to give some consideration usefully in rethinking the approach in this field. (Only for bases research intent). This is not a systematic review but the same the results of finalized research about new methods to be verified.

\section{Results}

\section{In scientific literature we can find}

Nakahara et al., write: Vascular calcification is a hallmark of atherosclerosis. The location, density, and confluence of calcification may change portions of the arterial conduit to a noncompliant structure. Calcifications may also seed the cap of a thin cap fibro atheroma, altering tensile forces on the cap and rendering the lesion prone to rupture. Many local and systemic factors participate in this process, including hyperlipidemia, ongoing inflammation, large necrotic cores, and diabetes. Vascular cells can undergo chondrogenic or osteogenic differentiation, causing mineralization of membranous bone and formation of endochondral bone. Calcifying vascular cells 
are derived from local smooth muscle cells and circulating hematopoietic stem cells (especially in intimal calcification). Matrix vesicles in the extracellular space of the necrotic core serve as a nidus for calcification. Although coronary calcification is a marker of coronary atheroma, dense calcification ( $>400 \mathrm{HU}$ ) is usually associated with stable plaques. Conversely, micro calcification (often also referred to as spotty calcification) is more commonly an accompaniment of vulnerable plaques. Recent studies have suggested that micro calcification in the fibrous cap may increase local tissue stress (depending on the proximity of one micro calcific locus to another, and the orientation of the micro calcification in reference to blood flow), resulting in plaque instability [1].

According Romer et al., Coronary intravascular ultrasound (IVUS) can assess arterial wall architecture and localize large intravascular deposits, but it does not provide quantitative chemical information, which is essential in the evaluation of atherosclerotic lesions. Previously, it has been shown that Raman spectroscopy can be used to accurately quantify the relative weights of cholesterol, calcium salts, triglycerides, and phospholipids in homogenized arterial tissue. The combination of Raman spectroscopy and IVUS applied in vitro provides detailed information about the amount and location of calcific deposits and lipid pools in atherosclerotic plaques. Future advances in optical fiber technology may allow simultaneous collection of Raman spectra and IVUS images through the same catheter in vivo [2].

Ranade V write that: In comparison with many of the other drug delivery systems, implantable pumps and implants for variable rate delivery are at a crude stage of development. Although exceptions exist, the typical implantable pump consists of an electromechanically complex mechanism to regulate drug delivery from a percutaneous refillable reservoir, while power to drive the system comes from a transcutaneous energy transmission system. The potential for electrical or mechanical failure is high, and the systems are not yet sufficiently convenient or easy to use to recommend in a routine therapy. Problems with refilling of an apparently well designed implanted reservoir have been observed while, at the same time, cutaneous energy transmission systems are not well established. In most instances, the development of an elementary osmotic pump system dosage form follows a well-defined path of physical-chemical formulation and clinical testing. The benefits most often provided by the dosage form are expected to be increase in selectivity of drug action achieved by the system's zeroorder release rate, and decrease in frequency of administration. The success in achieving these values is quantifiable from the pharmacology of the drug substance and its pharmacokinetics. Osmotic and other technical approaches to producing economical, rate-controlled dosage forms will make it possible for all new pharmaceutical products to carry kinetic specification of rate as well as static specification of content [3].

According et al. hou H: Implantable drug delivery systems (DDS) provide a platform for sustained release of therapeutic agents over a period of weeks to months and sometimes years. Such strategies are typically used clinically to increase patient compliance by replacing frequent administration of drugs such as contraceptives and hormones to maintain plasma concentration within the therapeutic window. Implantable or injectable systems have also been investigated as a means of local drug administration which favors high drug concentration at a site of interest, such as a tumor, while reducing systemic drug exposure to minimize unwanted side effects. Significant advances in the field of local DDS have led to increasingly sophisticated technology with new challenges including quantification of local and systemic pharmacokinetics and implant-body interactions. Because many of these sought-after parameters are highly dependent on the tissue properties at the implantation site, and rarely represented adequately with in vitro models, new nondestructive techniques that can be used to study implants in situ are highly desirable. Versatile imaging tools can meet this need and provide quantitative data on morphological and functional aspects of implantable systems [4]. 
Maniscalco et al., showed that: Atherosclerosis is a complex process with multiple mechanisms and factors contributing to its initiation and progression. Detection and quantification of coronary artery calcium (CAC) scores with electron beam tomography has been shown to correlate with obstructive and non-obstructive coronary artery disease (CAD). Pathogen-triggered calcification could play a role in CAD. Recent reports suggest that infectious blood Nano bacteria (NB) emerge to be such a trigger. So far, minimal or no reversal of atherosclerosis has been claimed by therapies with iv ethylenediaminetetraacetic acid disodium salt (EDTA), antibiotics, or other regimens, and therapies for atherosclerosis remain non-curative. We have now combined EDTA with antibiotic tetracycline (comET), an in vitro proven nanobacteriocidal treatment, and tested comET therapy in patients with documented CAD. Three hypotheses were probed: (1) Are NB present in patients with CAD?; (2) Does treatment with comET affect blood NB antigen and serology?; (3) Does a comET decrease CAC scores? One hundred patients with stable CAD and positive CAC scores were enrolled into a 4 month study of comET therapy. ComET therapy is composed of (1) Nutraceutical Powder (Vitamin C, Vitamin B6, Niacin, Folic Acid, Selenium, EDTA, l-Arginine, l-Lysine, l-Ornithine, Bromelain, Trypsin, CoQ10, Grapeseed Extract, Hawthorn Berry, Papain) $5 \mathrm{~cm}$ (3) taken orally every evening; (2) Tetracycline $\mathrm{HCl}$ $500 \mathrm{mg}$ taken orally every evening; (3) EDTA $1500 \mathrm{mg}$ taken in a rectal suppository base every evening. CAC scoring was repeated at 4 months and serum samples were analyzed for NB antigen and serology at baseline, 2 and 4 months. Complete blood count, metabolic panel, liver function, C-reactive protein (hs-CRP) and lipids were analyzed at baseline and 4 months. Seventy-seven patients completed the study and all patients were positive for NB serology, antigen or both. Responders $(n=44 ; 57 \%)$ had significant decreases in total CAC scores $(\mathrm{P}=0.001)$, the average decrease being $14 \%$. Non-responders $(n=33 ; 44 \%)$ had no change or had increases in CAC scores. Angina was decreased or ablated in 16 of 19 patients (84\%). Lipid profiles improved to non-atherogenic direction significantly $(\mathrm{P}=0.001)$, a remarkable finding in a patient group where $86 \%$ were on continuous statin medication already before the trial. In conclusion, CAC scores decreased during ComET therapy trial in most CAD patients inferring regression of calcified coronary artery plaque volume. The patients tolerated the therapy well and their angina and lipid profiles improved [5].

And by Gervasio A, et al.: Ethylene diamine tetraacetic acid (disodium EDTA or edetate disodium), patented in 1938 by Munz, is a chelating agent capable of binding cationic metallic and nonmetallic ions and mobilizing them from physiological tissue in a process termed chelation. EDTA can complex with metals such as lead and cadmium, with the EDTA-metal chelate being excreted in the urine. Edetate disodium (the sodium salt of EDTA), which binds to calcium among other cations, was first used to treat hypercalcemia and digitalis intoxication. Later, in the mid-20th century, motivated by the calcification present in advanced coronary disease, practitioners began using edetate disodium to treat symptoms of cardiovascular disease with initially positive, albeit uncontrolled, results. These early reports were followed by over 50 years of uncontrolled case reports and case series 3 and 3 small clinical trials. The trials enrolled fewer than 300 patients in aggregate and had surrogate endpoints and short follow-up. Although interpreted as negative, they could not exclude a smallto-moderate benefit of edetate disodium chelation therapy. Despite, or because of, an absence of clear evidence, most traditional physicians shunned the practice.

Despite chelation therapy being rejected by traditional medicine, pockets of practitioners continued administering edetate disodium treatments with many case reports and case series published. Occasionally, there were reports of serious harm, including death, that were usually due to errors in drug selection, dosage, or rate of infusion. To definitively determine the effectiveness and safety of chelation therapy, the National Center for Complementary and Alternative Medicine and the National Heart, 
Lung, and Blood Institute released a request for applications in 2001 for a definitive study of edetate disodium treatment in subjects with coronary artery disease (CAD), and the aptly named TACT (Trial to Assess Chelation Therapy) was born.

TACT patients were aged 50 years or older and had an acute myocardial infarction (MI) at least 6 weeks prior to enrollment and a creatinine level of $2.0 \mathrm{mg} / \mathrm{dL}$ or less.8 Because chelation therapy was typically administered in conjunction with a highdose oral multivitamin and multimineral regimen, a $2 \times 2$ factorial trial was designed to test intravenous chelation versus intravenous placebo and oral multivitamin and multimineral versus placebo vitamins/minerals. The primary composite endpoint was all-cause mortality, MI, stroke, coronary revascularization, and hospitalization for angina, which is similar to that used in the ACCELERATE (Assessment of Clinical Effects of Cholesteryl Ester Transfer Protein Inhibition With Evacetrapib in Patients at a High-Risk for Vascular Outcomes), WIZARD (Weekly Intervention with Zithromax for Atherosclerosis and its Related Disorders), and JUPITER (Justification for the Use of Statins in Primary Prevention: An Intervention Trial Evaluating Rosuvastatin) trials and other modern studies. A total of 1708 patients were enrolled with a median followup of 55 months. Patients were treated with 40 infusions containing up to 3 grams of intravenous edetate disodium (reduced based on renal function).

The results of TACT, which were widely expected to drive the final nail into the coffin for chelation therapy, surprised the blinded investigators and shocked the traditional medical community. Chelation therapy reduced the risk of cardiovascular events by $18 \%$ (hazard ratio $[\mathrm{HR}]=0.82$; $95 \%$ confidence interval [CI], 0.69-0.99; $\mathrm{p}=0.035$ ), with a 5 -year number needed to treat (NNT) of 18 patients to prevent an event. Continued separation between the two curves at the completion of the study suggested a sustained effect of toxic metal removal by chelation.

Diabetes mellitus was prespecified as a subgroup for analysis in TACT and consisted of a total of 633 (37.1\%) patients. Treatment with EDTA infusions reduced the primary endpoint (EDTA chelation vs. placebo: $25 \%$ vs. $38 \%$; $\mathrm{HR}=0.59 ; 95 \% \mathrm{CI}$, $0.44-0.79 ; \mathrm{p}=0.0002$ ).There was a $15 \%$ absolute decrease in the 5 -year Kaplan-Meier primary event rate. The 5-year NNT was 6.5 (95\% CI, 4.4-12.7). Rates of the principal secondary endpoint (a composite of cardiovascular disease death, MI, and stroke) were also lower for diabetic patients randomized to EDTA chelation (HR=0.60; 95\% CI, 0.39$0.91 ; \mathrm{p}=0.017$ ), with a $5.1 \%$ absolute decrease in the 5 -year Kaplan-Meier event rate. Chelation also reduced total mortality in the diabetes subgroup (HR=0.57; 95\% CI, $0.36-0.88 ; p=0.011 ; 5$-year NNT=12). In addition, there was a significant reduction in recurrent MI (HR=0.48; 95\% CI, 0.26-0.88; $\mathrm{p}=0.015$ ) and coronary revascularizations (HR=0.68; 95\% CI, 0.47-0.99; $\mathrm{p}=0.042$ ). One other notable feature of TACT was the long-term pattern of benefit. Some prognosis-modifying treatments, such as coronary artery bypass graft for advanced CAD, are applied once and typically show an amplifying benefit after therapy. Most medical secondary prevention treatments used in CAD, however, need to be continued in order to provide sustained protection from events. This was not true for chelation in TACT. The event rate curves continued to separate after the infusions ended, and that unusual treatment effect pattern may be a clue to the mechanism of action, such as depletion of a toxicant, such as lead or cadmium that has accumulated in the body over many years.

Recognizing the weight of the TACT results, the American Heart Association and the American College of Cardiology upgraded edetate disodium chelation from a $3 \mathrm{C}$ to a $2 \mathrm{~B}$ indication in their 2014 revision of the guidelines for the treatment of chronic ischemic heart disease [6].

Maria D. Avila et al. write that: The trial to assess chelation therapy was a $\$ 30$ million National Institutes of Health-funded study of the safety and efficacy of EDTA- 
based chelation infusions in 1708 post-myocardial infarction (MI) patients. The trial to assess chelation therapy demonstrated a significant $(\mathrm{P}=0.035) 18 \%$ reduction in a combined primary endpoint of death, MI, stroke, coronary revascularization, or hospitalization for angina. In diabetic patients the benefit was more extreme, with a $41 \%$ relative reduction in risk $(\mathrm{P}=0.0002)$ and a $43 \%$ reduction in total mortality $(\mathrm{P}=0.011)$. Safety data were favorable. A reduction of oxidative stress by chelation of toxic metals has been proposed as a possible mechanism of action [7].

"The clinical pharmacist specific competences added to medicinal chemistry knowledge and clinical need are efficacy instruments to translate to pharmaceutical industries the modification of pharmacological molecule or the need to search new strategy in drug therapy if not efficacy as request [8]."

\section{Discussion}

Related to the bibliography reported and other in biomedical database available today. We have seen that in many situation pharmacological molecules are added to other substrate in order to improve the effect in local site, reducing global side effect. The same we have seen that some interventional radiology teqniques have been used to avoid general classic surgery with good clinical results as we can see in biomedical databases and scientific library. Observing the composition of calcified aortic valves we can think that a new system that can be able to dissolve the salt precipitation can be used to improve the valve phisiology versus a complex classic surgery. Is universally known that the patient health highly is involved in artery phisio-pathology and that cardiovascular disease are responsible of a great number of preventable exitus. Using the association of interventistic radiology strategy to inject in valve tissue a mixture of pharmacological agent (complexant or other) with a delivery system designed to improve the time of action in site we can think to obtain an relevant effect to be considered (starting from animal models). So we can search a strategy systems to make possible the pharmacological molecules can arrive in the Site interested by the pathology avoiding the global toxicology of some drugs (as EDTA). In example we can see that commonly surgery is used in aortic valvle disease in order to prevent $\mathrm{CHF}$ and re modulations of cardiac tissue. This imply a complex surgery situation and to find other solution can be an interesting option. We have seen that vascular calcification is heavly involved in atherosclerotic disease and that this phenomena can be better showed also by tomography and RAMAN SPECTROSCOPY.

And that using innovative drugs delivery systems we can improve clinical effect reducing total toxicity. And even if the chelanting therapy is not accepted by scientific world [9] we must say that this could be related to a systemic sub ministration with global toxicity, and not related to local treatment. And we can see that avila et al write that TACT, the first large-scale trial of chelation therapy for atherosclerotic coronary disease, found that EDTA chelation therapy reduced the risk of a composite of adverse cardiovascular outcomes, particularly among patients with diabetes. Before disodium EDTA chelation can take its place among other accepted therapies in the routine care of post-MI patients, however, it is important that further replicative and mechanistic clinical trials be performed [10].

According King SB et al.: Different strategies can be applied in some coronaric disease with different profile of results and side effect: Coronary artery disease (CAD) is one manifestation of ischemic heart disease, which is the leading cause of mortality in the world. In addition to preventive medical therapy and lifestyle changes, consideration of revascularization of obstructed arteries to reduce ischemia, alleviate angina, and improve quality of life is a mainstay of current practice. However, the benefits of different methods of revascularization in particular patient populations are debated. Percutaneous coronary intervention (PCI), which involves placement of intracoronary stents in most patients, is a less invasive procedure than coronary 
artery bypass graft (CABG) surgery. Although it is generally accepted that patients with single-vessel obstructive CAD are best treated with PCI, patients with multivessel CAD have a higher ischemia burden, a greater risk for developing recurrent ischemic events, and a higher mortality. It is in this patient population where the debate over revascularization with stents versus surgery continues [11].

In order to dissolve the atherosclerotic plaques precipitated on cardiac vales, what we can think to physical system, inject inside the vascular wall a mixture of a polymerbased extended

\section{Release delivery system}

- With a chelating agent will eat up the cationic precipitations if it is a sal such as calcium containing material

- Or we can use steroid agents if it is a cholesterol-based we can use a type of material that will soften the plaques because of the substitution of cholesterol with another sterole like sitosterol or other molecules with this kind of activities.

\section{Conclusion}

Even if the results showed in this paper are not conclusive and that specific more experimental data are necessary we can say that Under the light of the article finded in this work but also to other works published We can think a new system "to regenerate" a valve tissue calcified. We think that adding 3 strategies we can have relevant effect. We have seen that atherosclerotic plaques has calcific components and that EDTA is a calcium complexant agent and we have also see that interventional radiology strategy help physicians to transfer in example contrast agent in situ or medicated stents We can think that adding this 3 strategies we can have more clinical outcomes and less complex toxicity if used active but toxic molecule as EDTA AS CALCIUM COMPLESSANT In the reverse atherosclerotic plaques. Using a toxic agent but in local way we think we can obtain more useful result. A chemistry modify make possible to link the EDTA molecule to a group with easily renal clearance. Even if in past systemic therapy has not showed a real activity a new delivery systems could improve the final results. The same we can consider a new injection system to deliver the drugs into the valve tissue.

\section{Declaration}

This article has not diagnostics or therapy intent only to produce research hypothesis. Any ethical consideration must be evalued (animal model and other level) by next researcher interested in.

\section{References}

1. Nakahara T, Dweck MR, Narula N, Pisapia D, Narula J, et al. Coronary Artery Calcification: From Mechanism to Molecular Imaging. JACC Cardiovasc Imaging. 2017; 10: 582-593. Ref.: https://goo.gl/jDdACD

2. Römer TJ, Brennan JF, Puppels GJ, Zwinderman AH, van Duinen SG, et al. Intravascular ultrasound combined with Raman spectroscopy to localize and quantify cholesterol and calcium salts in atherosclerotic coronary arteries. Arterioscler Thromb Vasc Biol. 2000; 20: 478-483. Ref.: https://goo.gl/VzXgbw

3. Ranade VV. Drug delivery systems. 4. Implants in drug delivery. J Clin Pharmacol. 1990; 30: 871889. Ref.: https://goo.gl/bcaszv

4. Zhou H, Hernandez C, Goss M, Gawlik A, Exner AA. Biomedical Imaging in Implantable Drug Delivery Systems. Curr Drug Targets. 2015; 16: 672-682. Ref.: https://goo.gl/Hxup88

5. Maniscalco BS, Taylor KA. Calcification in coronary artery disease can be reversed by EDTAtetracycline long-term chemotherapy. 2004; 11: 95-101. Ref.: https://goo.gl/GRwvzW

6. Lamas GA, FACC MD, Ian Ergui BS, Omar M Issa. Chelation Therapy for CAD. DO Expert Analysis. 2016. Ref.: https://goo.gl/WP8UWY 
7. Avila MD, Escolar E, Lamas GA. Chelation therapy after the Trial to Assess Chelation Therapy: results of a unique trial. Curr Opin Cardiol. 2014; 29: 481-488. Ref.: https://goo.gl/TehvBS

8. Luisetto M, Nili-Ahmadabadi B. The Clinical Pharmacist Competence as Pharmaceutical Drug Design Tool. Research \& Reviews: Journal of Hospital and Clinical Pharmacy. 2017; 3: 1-2. Ref.: https://goo.gl/D1sptg

9. Ernst E. Chelation therapy for coronary heart disease: An overview of all clinical investigations. Am Heart J. 2000; 140: 139-141. Ref.: https://goo.gl/QYK9vn

10. Avila MD, Escolar E, Lamas GA. Chelation therapy after the Trial to Assess Chelation Therapy: results of a unique trial. Curr Opin Cardiol. 2014; 29: 481-488. Ref.: https://goo.gl/Vk9bkr

11. King SB, Marshall JJ, Tummala PE. Revascularization for coronary artery disease: stents versus bypass surgery. Annu Rev Med. 2010; 61: 199-213. Ref.: https://goo.gl/uhUc2d 